\title{
Tuberculosis in Nepal: Diagnosis and Treatment Barriers
}

\author{
SB Marahatta ${ }^{1-3}$, BJ Karki \\ ${ }^{1}$ Editor JMMIHS, ${ }^{1}$ WHO-TDR Post Doc Research Fellow ${ }^{2}$ \\ ${ }^{3}$ Associate Professor, Manmohan Memorial Institute of Health Sciences, IOM-TU \\ ${ }^{4}$ MBBS Universal College Medical Sciences Nepal
}

Tuberculosis (TB) is a major public health concern for Nepal like many other developing countries around the world. The incidence of TB in Nepal according to World Bank is 158 per 100,000 people. On the other hand, the case detection rate for all forms of TB in Nepal is only about 79\% [1]. The cases that are not notified and detected are not enrolled for Directly Observed Treatment Short course (DOTS) therapy. They do not get adequate treatment and remain infectious, increasing the TB affected patient load of the country.

Treatment of TB is free of cost by Government of Nepal. DOTS are composed of five distinct elements: political commitment; microscopy services; drug supplies; surveillance and monitoring systems and use of highly efficacious regimens; and direct observation of treatment. Government provides DOTS service in Nepal through DOTS treatment centers and sub-centers.

A simple rule is that early diagnosis of TB leads to early treatment. As the treatment gets delayed, the severity of the disease and difficulty in recovery process becomes complicated. As being one of the major public health problems in Nepal, halting and decreasing TB infection faces add-on challenge with the advent of co-infection and possibility of increase in drug resistant TB [2]. This is why detecting TB in its early stage would pose a number of advantages to the patient that would in turn help for early treatment. However, the challenges and barriers in early diagnosis remain that is being contributed by multifaceted factors.

On study showed that in Nepal patients resort to number of providers and institutions before being diagnosed properly for TB [3]. In addition, they spent long period of time (sometime years) before being diagnosed properly. This is specifically due to the fact that patients usually resort to institutions or facilities that lack proper arrangement for TB diagnosis such as pharmacies and informal health care settings. The lack of knowledge coupled with poor financial capacity to pay for the diagnosis [4] especially in rural population prevents them from being diagnosed properly as well.

Moreover, the disease (TB) could mimic other diseases making the case typical or atypical which in turn make it difficult for physicians and health workers in its proper diagnosis [5]. Even in co-infection, such as with HIV, occurrence of TB could be a difficulty creating diagnostic complacency. Extra pulmonary TB is another state where its diagnosis can be missed as it manifests in other organs or sites of the body [6, 7]. In many of the cases, the case of TB is also overlooked by the physicians that are mostly being attributed to lack of diagnostic facilities, inadequate investigation of the ailment and physician's specialization [8].

Given the complexity of the disease, Nepal remains ill prepared to tackle the issues related with early diagnosis of TB. From the lack of preparedness of health system especially in rural areas, poor socioeconomic status of majority of the people, to the lack of awareness and knowledge regarding TB [9] might pose grave challenge in diagnosing TB at early stage.

TB treatment in Nepal is through DOTS. It is a treatment course where patient has to comply with the treatment regimen continuously for a period of time, generally 6 months. However, availability of treatment facility does not necessarily mean that it has been accessed and utilized in proper manner. Treatment aspect of TB has been related with number of issues that need to be addressed to improve 
its compliance and outcome.

Socioeconomic issues and stigmas are related to the disease. There is problem in understanding of the regimens and being complaint to the services provided [10] that also reflects the provider-patient relation as one of the important factors in treatment. Patients find it difficult in seeking T.B treatment as they don't trust the program and most of them don't possess enough knowledge about the disease itself [4]. In addition, the policies regarding the adoption of treatment should be contextual [11]. Female patient shows lowest compliance due to financial barriers and afraid of being socially isolated. According to T.B staff differences in urban and rural settings was high number of patients and difficulty in providing services in hard to reach area respectively. Migration from rural to urban areas or one country to another is another factor [9]. Then, there is the matter of diagnosis of the disease that has been discussed in the previous section of this paper. All of these factors related to socio-cultural and socio-economic condition, knowledge, geographical setting; contribute to treatment default.

In conclusion, TB still remains a major threat in Nepal; especially given its complex construct coupled with diverse individual, societal and environmental factors that serve as favorable platform for the disease to prevail in Nepalese population. There is need for better response from Government to regular supply side especially in private and informal sector including enhancing focus on improving knowledge and awareness regarding timely TB diagnosis and treatment.

\section{References}

1. World Bank. $2014 . \quad$ Indicators. Retrieved from http://data.worldbank.org/indicator/SH.TBS.DTEC.ZS

2. Teo, S. K. Tuberculosis - Barriers to Early Diagnosis. Singapore Med J 2002;43(4):169.

3. Asbroek AHA, Bijlsma MW, Malla, P, Shrestha B and Delnoij DMJ. The road to tuberculosis treatment in rural Nepal: A qualitative assessment of 26 journeys. BMC Health Services Research 2008;8:7

4. Long Q, Li Y, Wang Y, Yue $Y$, Tang C, Tang S, Squire SB and Tolhurst R. Barriers to accessing TB diagnosis for rural-to-urban migrants with chronic cough in Chongqing, China: A mixed methods study 2008; BMC Health Services Research 2008, 8:202 doi:10.1186/14726963-8-202

5. Lerner $\mathrm{BH}$. Revisiting the death of Eleanor Roosevelt: was the diagnosis of tuberculosis missed? Int J Tuberc Lung Dis 2001; 5(12):1080-85.

6. Katz I, Rosental MD and Michaeli D. Undiagnosed tuberculosis in hospitalised patients. Chest 1985; 87:770-74.

7. Rowinska-Zakrzewska, Szopinski J, Remiszewski, P, Szymanska D, Miller M, Pawlicka L. and Zwolska-Kwiek. Tuberculosis in the autopsy material: analysis of 1500 autopsies performed between 1972 and 1991 in the Institute of Tuberculosis and Chest Diseases, Warsaw, Poland. Tubercle Lung Dis 1995;76:349-54.

8. Liam CK and Tang BG. Delay in the diagnosis and treatment of pulmonary tuberculosis in patients attending a university teaching hospital. Int J Tuberc Lung Dis 1997;1(4):326-32

9. Harper $M$, Ahmadu FA, Ogden JA, McAdam KP and Lienhardt C. Identifying the determinants of tuberculosis control in resource-poor countries: insights from a qualitative study in The Gambia. Transactions of the Royal Society of Tropical Medicine and Hygiene 2003;97:506-10.

10. Noyes J and Popay J. Directly observed therapy and tuberculosis: how can a systematic review of qualitative research contribute to improving services? A qualitative meta-synthesis. Journal of Advanced Nursing 2007;57(3): 227-43.

11. Harper I. National Tuberculosis Control Programmes of Nepal and India: Are They Using the Correct Treatment Regimens? Journal of Health Studies. 2009;2:51-67 